\title{
OBSERVATIONS OF MAGNETIC HELICITY
}

\author{
L. van Driel-Gesztelyi $1,2,3,4$, P. Démoulin ${ }^{3}$, and C.H. Mandrini ${ }^{5}$ \\ ${ }^{1}$ Centre for Plasma Astrophysics, K.U. Leuven, Celestijnenlaan 200B, 3001 Heverlee, Belgium \\ ${ }^{2}$ Mullard Space Science Laboratory, Univ. College London, UK \\ ${ }^{3}$ Observatoire de Paris, LESIA, FRE 2461 (CNRS), Meudon Cedex, France \\ ${ }^{4}$ Konkoly Observatory of the Hungarian Academy of Sciences, Budapest, Hungary \\ ${ }^{5}$ Instituto de Astronomía y Física del Espacio, IAFE, Argentina
}

\begin{abstract}
The first observational signature of magnetic helicity in the solar atmosphere (sunspot whirls) was discovered 77 years ago. Since then, the existence of a cycle-invariant hemispheric helicity pattern has been firmly established through current helicity and morphological studies. During the last years, attempts were made to estimate/measure magnetic helicity from solar and interplanetary observations. Magnetic helicity (unlike current helicity) is one of the few global quantities that is conserved even in resistive magnetohydrodynamics (MHD) on a timescale less than the global diffusion timescale, thus magnetic helicity studies make it possible to trace helicity as it emerges from the sub-photospheric layers to the corona and then is ejected via coronal mass ejections (CMEs) into the interplanetary space reaching the Earth in a magnetic cloud. We give an overview of observational studies on the relative importance of different sources of magnetic helicity, i.e. whether photospheric plasma motions (photospheric differential rotation and localized shearing motions) or the twist of the emerging flux tubes created under the photosphere (presumably by the radial shear in the differential rotation in the tachocline) is the dominant helicity source. We examine the sources of errors present in these early results and try to judge how realistic they are.
\end{abstract}

\section{INTRODUCTION}

In 1925, G.E. Hale published a two-page paper in PASP describing vortices seen in $\mathrm{H} \alpha$ around sunspots, what he called sunspot whirls. Investigating data extending over three solar cycles he found no relationship between the direction of these vortices and spot polarity and found no reversal of the whirl direction together with the general reversal of spot polarities at cycle changes. He found a cycle-invariant rule: about $80 \%$ of sunspot whirls are counter-clockwise on the northern hemisphere and clockwise in the south. Drawing a parallel with the hemispheric rule known for terrestrial cyclones, he concluded that these whirls are hydrodynamical rather than electromagnetic phenomena, i.e. they are related to the solar rotation. Richardson (1941) repeated the investigation on data extending over four solar cycles and confirmed Hale's results. However, he also pointed out that whirls are observed only around $16 \%$ (32\% if he made allowances for defective plates and poor seeing) of the sunspots which are sufficiently large ( $\geq 200 \mathrm{MSH}$ [millionth of the solar hemisphere]) to show them clearly in the Mt. Wilson plates.

The subject was awaken in the 90s, when hemispheric chirality or handedness patterns independent of sunspot cycle were discovered in active regions, coronal loops, filaments, coronal arcades and interplanetary magnetic clouds (MCs) (Leroy et al., 1983; Seehafer, 1990; Pevtsov et al., 1995; Abramenko et al., 1996; Martin et al., 1994; Rust, 1994, Martin and McAllister, 1997; Rust and Kumar, 1996; Canfield et al., 1999; for a most recent review see paper by Pevtsov, 2003, and references therein). A quantitative, mathematical measure of the chiral properties of these structures is the magnetic helicity (Berger and Field 1984; Berger, 
1999). These chirality patterns indicate that the Sun preferentially exhibits left-handed features in its northern hemisphere and right-handed features in the south. A right-handed twist and a clockwise rotation of the loops when viewed from above implies positive helicity, and vice versa for negative helicity, so the recent results agree with those of Hale and Richardson. However, it is noteworthy that in the formation of sunspot whirls, besides or instead of helicity (Nakagawa et al., 1971), the Coriolis force acting in the Evershed flow and its interaction with the magnetic field (Peter, 1996) can play an important role.

Exceptions to these helicity rules occur in most categories of solar activity at a significant percentage (20-35\%). Nevertheless, the Sun's preference for features adhering to these rules motivates the search for an underlying mechanism that is, evidently, global in scope. Where and by what kind of mechanism(s) is magnetic helicity generated? The main candidates are the dynamo, helical turbulence (the $\alpha$ or the $\Sigma$ effect) and differential rotation (both sub-surface and photospheric, the $\Omega$ effect). However, theoretical works analyzing the efficiency and even ability of some of these mechanisms to create the observed dominant hemispheric helicity pattern have produced highly controversial results (Seehafer, 1990, 2003; Brandenburg and Blackman, 2003; Longcope and Pevtsov, 2003; Berger and Ruzmaikin, 2000; DeVore, 2000; Démoulin et al., 2002a).

When theory is producing confusing results the task for observers is to do their best in providing constraints for models. The appearance of quantitative observational works on magnetic helicity has been a very recent development in solar physics. We make an attempt below to review these papers and try to judge how realistic the present results are.

\section{THE IMPORTANCE OF MAGNETIC HELICITY STUDIES}

Magnetic helicity quantifies how the magnetic field is sheared or twisted compared to its lowest energy state (potential field). Observations provide plenty of evidence for the existence of such stresses in the solar magnetic field and their association to e.g. flare and CME activity, but its precise role in such activity events is far from being clear.

Magnetic helicity is one of the few global quantities, which is conserved even in resistive MHD on a timescale less than the global diffusion timescale (Berger, 1984). Thus, as magnetic flux travels from the tachocline through the convection zone, emerges through the photosphere into the corona and is ejected into the interplanetary space during CME events, the magnetic helicity it carries can be traced.

\section{COMPARISON OF MAGNETIC AND CURRENT HELICITIES}

Note, that magnetic helicity is different from current helicity, which has been extensively used in establishing the hemispheric helicity rules. Magnetic helicity is defined by a volume integral: $H_{m}=\int_{V} \vec{A} \cdot \vec{B} d V$, where $\vec{A}$ is the magnetic vector potential, $\vec{B}=\vec{\nabla} \times \vec{A}$ is the magnetic field, while the definition of current helicity is: $H_{c}=\int_{V} \vec{B} \cdot \vec{j} d V$, with $\mu_{0} \vec{j}=\vec{\nabla} \times \vec{B}$. While $H_{m}$ is, in general, gauge dependent through $\vec{A}$ (see next Section), there is no gauge freedom with $H_{c}(\vec{\nabla} \cdot \vec{B}=0)$. Furthermore, while $H_{m}$ is a conserved MHD quantity, this is not the case for $H_{c}$ ! However, it is usually true, that $H_{m}$ and $H_{c}$ have the same sign.

Furthermore, we should be aware of the fact, that observationally derived values of $H_{c}$ represent only a fraction of its full value. The volume integral can be written out with its components: $H_{c}=\int_{V} \vec{B} \cdot \vec{j} d V=$ $\int_{V}\left(B_{x} \cdot j_{x}+B_{y} \cdot j_{y}+B_{z} \cdot j_{z}\right) d V$, of which only the last component can be deduced from observations. Though using photospheric vector magnetograms all three components of $\vec{B}$ are available, only the vertical component of $\vec{j}$ can be computed (via the horizontal derivatives of $\vec{B}$ ). Thus, what we can observationally determine as current helicity is merely the following surface integral: $h_{c}=\int_{\text {photosphere }} B_{z} \cdot j_{z} d S$.

The general relationship between magnetic and current helicities are not known, however, they both are commonly regarded as proxies for twist in magnetic fields.

\section{RELATIVE MAGNETIC HELICITY}

Computation of magnetic helicity $H_{m}=\int_{V} \vec{A} \cdot \vec{B} d V$ is physically meaningful only when $\vec{B}$ is fully contained inside the volume V. However, when this is not so (when the normal component $B_{n} \neq 0$ along the boundary 
S), following Berger and Field (1984), a relative magnetic helicity, which is gauge independent, can be computed by subtracting the helicity of a reference field $B_{0}$, which has the same $B_{n}$ distribution on $\mathrm{S}$ as $\vec{B}: \quad H_{r}=\int_{V} \vec{A} \cdot \vec{B} d V-\int_{V} \overrightarrow{A_{0}} \cdot \overrightarrow{B_{0}} d V$, with $\overrightarrow{A_{0}}$ satisfying $\overrightarrow{B_{0}}=\vec{\nabla} \times \overrightarrow{A_{0}}$. Since $H_{r}$ is well conserved under solar conditions, the only way helicity can be modified inside $V$ is by helicity flux crossing the boundary $\mathrm{S}$ (Berger and Field, 1984): $\frac{d H_{r}}{d t}=-2 \int_{S}\left[\left(\overrightarrow{A_{0}} \cdot \vec{v}\right) \vec{B}-\left(\overrightarrow{A_{0}} \cdot \vec{B}\right) \vec{v}\right] \cdot \overrightarrow{d S}$, where $\vec{v}$ is the velocity of the plasma. The $1^{\text {st }}$ term corresponds to helicity flux by footpoint motions parallel to $\mathrm{S}$ (shear term), while the $2^{\text {nd }}$ term denotes inflow and outflow of helicity through the boundary S (advection term).

The shear term involves helicity injection by footpoint motions in the photosphere like twisting, shearing (special case: differential rotation) and braiding, while the advection term includes emerging motions (but also submergence!) through the photospheric boundary as well as ejection of helicity via CMEs through the coronal boundary of the magnetic volume.

\section{QUANTITATIVE OBSERVATIONAL ANALYZES OF MAGNETIC HELICITY}

\section{Magnetic Helicity Injected by Differential Rotation}

For the computation of magnetic helicity injected by photospheric plasma motions Berger (1984, 1988) derived an expression for $d H_{r} / d t$ which depends only on observable photospheric quantities $\left(B_{n}\right.$ and $\left.\vec{v}\right)$. DeVore (2000) calculated magnetic helicity injection by differential rotation and found that this timeindependent shearing flow, surprisingly, does not provide a monotonous input of magnetic helicity (Figure 1). Berger (1986) showed that the helicity injection rate can be understood as the summation of the rotation rate of all the individual elementary flux pairs weighted by their magnetic flux: $d H_{r} / d t=-\frac{1}{\pi} \int_{S} \int_{S^{\prime}} \vec{R} \times$ $\vec{v}(\vec{r}) / R^{2}{ }_{n} B_{n}(\vec{r}) B_{n}\left(\overrightarrow{r^{\prime}}\right) d S . d S^{\prime}$, where $\vec{R}=\vec{r}-\overrightarrow{r^{\prime}}$ is the difference in spatial positions on the photospheric plane. Using this result, Démoulin et al. (2002b) have shown that horizontal photospheric plasma motions imply two different helicity terms: the rotation of each polarity introduces 'twist' helicity, while the relative rotation of opposite polarity flux concentrations injects 'writhe' helicity: $\Delta H_{r}(t)=\left.\Delta H_{r}(t)\right|_{\text {twist }}+\left.\Delta H_{r}(t)\right|_{\text {writhe }}$. In case of a shear-flow like the differential rotation, the twist and writhe helicity fluxes always have opposite signs, while their magnitudes are similar, thus they partially cancel. The relative importance of twist and writhe helicity injection by differential rotation depends mainly on the orientation of the bipole. Twist (writhe) helicity dominates when the bipole axis is parallel (perpendicular) to the equator; these two terms are of the same magnitude and therefore cancel for a bipole tilt of about $45^{\circ}$. As the tilt angle of a bipolar $\mathrm{AR}$ is changing due to the shearing effect of the differential rotation flow, the helicity injection rate is changing as well.

The evolution of the helicity injection rate in a typical AR (NOAA 7978, July-Nov. 1996), which was initially parallel to the equator and was only deformed by differential rotation, was computed by Démoulin et al. (2002a). In this south hemispheric AR differential rotation injected positive helicity, in accordance with the hemispheric helicity sign; however, the injection rate was low even initially and decreased with time. During six solar rotations differential rotation injected only a total of $8.3 \times 10^{42} M x^{2}$ which is about the maximum that it can provide to this AR.

Another study by Green at al. (2002) of a peculiar AR (NOAA 8100, Nov. 1997-Feb. 1998), in which the main polarities were rotating around one another by about $150^{\circ}$, showed a very different history of helicity injection. Since the AR, due to its rotation, had passed the critical $45^{\circ}$ orientation between its $2^{\text {nd }}$ and $3^{r d}$ rotations, helicity injection changed sign by the $3^{r d}$ rotation and became opposite (negative) to the dominant hemispheric helicity sign for the southern hemisphere (see Figure 6 in Green et al., 2002).

Due to the (partial) cancellation between twist and writhe helicities, photospheric differential rotation was shown to be very inefficient to provide magnetic helicity on the AR-scale (Démoulin et al., 2002a,b; Green et al., 2002; Nindos and Zhang, 2002). The maximum total helicity injected in a bipolar AR that is initially parallel to the equator having a magnetic flux $\Phi$ of $\approx 10^{22} \mathrm{Mx}$ is about $10^{43} \mathrm{Mx}^{2}$ (see Figure 5 in Démoulin et al., 2002b). Note that this value refers to helicity injected during the entire lifetime of an AR!

However, the conclusion is different in the convective zone for both a solar cycle and a global spatial scale. Berger and Ruzmaikin (2000) evaluated helicity generation by differential rotation using 22 years of magnetogram data (1976-1998) and differential rotation curves. They found that the helicity production in 

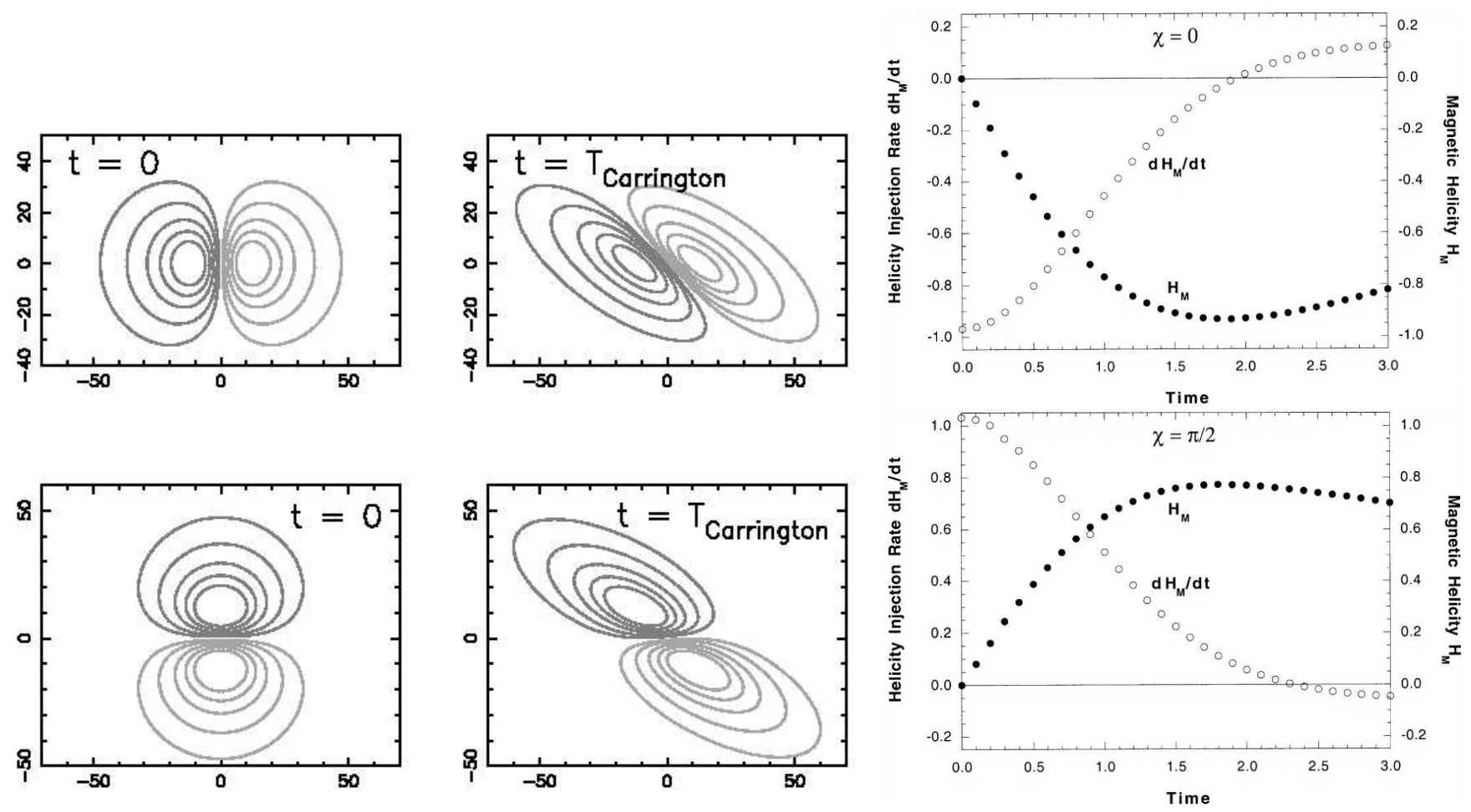

Fig. 1. Temporal evolution of a northern hemispheric bipole initially parallel (upper panels) and perpendicular to the equator (lower panels) deformed by differential rotation and the evolution of the relevant helicity injection rates (the latter graphs are used with permission, DeVore, 2000). Note that helicity injection by differential rotation, a time-independent shear flow, is not monotonous, due to the changing relative importance of twist and writhe helicities as the axis of the bipole is turning.

the interior by differential rotation had the correct sign compared to observations of coronal structures (the magnetic helicity conservation is satisfied by the natural generation of the same amount, but of opposite sign, in both hemispheres). The net helicity flow into each hemisphere over this 22-year magnetic cycle was found to be approximately $4 \times 10^{46} \mathrm{Mx}^{2}$.

\section{Helicity Injection by Photospheric Footpoint Motions}

Several authors computed helicity injection at the photospheric level by the shear term determining the transverse velocities of the magnetic flux concentrations in active regions by applying a local correlation tracking method (Chae 2001; Chae et al., 2002; Kusano et al., 2002; Moon et al., 2002; Nindos and Zhang, 2002). For these studies line-of-sight magnetic field maps of SOHO/MDI were used. Kusano et al., 2002, used also the transverse field measurements of the Solar Flare Telescope. To correct for foreshortening, a rotational mapping to the central meridian was applied. To determine $B_{n}$ from the longitudinal field the ARs were taken close to the centre disk. Furthermore, a small correction is applied to correct from the off disk centre position: it is generally assumed that magnetic fields in the photosphere are predominantly vertical.

The horizontal photospheric velocities are determined by tracking the evolution of magnetic flux tubes. The most widely used local correlation tracking (LCT) method was developed by November and Simon (1988). The photospheric velocities are determined by cross-correlating a small fraction of two subsequent images shifted with a variable spatial shift. The relative displacement is given by the highest correlation among the shifts, which provides an estimation for the velocities. Two important parameters in LCT have to be properly chosen: the FWHM (full width at half maximum), $w$, of the apodizing function and the time interval, $\Delta T$, between the two images to be compared. An optimal value of $w$ should be bigger than the size of the smallest features seen in the data, so it is related to the resolution of the magnetograms used. 
Table 1. Injection of magnetic helicity by photospheric footpoint motions and by differential rotation as obtained by correlation tracking in SOHO/MDI magnetograms. The left column is in units of $\mathrm{Mx}^{2}$ while the right column is in units of $\Phi^{2}$, where $\Phi$ is the magnetic flux of the AR (average of both polarities)

\begin{tabular}{|c|c|c|c|c|c|c|}
\hline \multirow{2}{*}{$\mathrm{AR}$} & \multirow{2}{*}{$\begin{array}{l}\text { Observing } \\
\text { period }(\mathrm{hr})\end{array}$} & \multicolumn{2}{|c|}{ Footpoint motions } & \multicolumn{2}{|c|}{ Differential rotation } & \multirow{2}{*}{ Reference } \\
\hline & & $10^{42} \mathrm{Mx}^{2}$ & $\Phi^{2}$ & $10^{42} \mathrm{Mx}^{2}$ & $\Phi^{2}$ & \\
\hline 8011 & 40 & 0.01 & 0.003 & 0.001 & 0.0003 & Chae (2001) \\
\hline 8100 & 6.5 & 1.0 & 0.006 & 0.09 & 0.0004 & Moon et al. (2002) \\
\hline & 120 & 4.0 & 0.02 & & & Kusano et al. (2002) \\
\hline 8668 & 50 & 2.9 & 0.03 & 0.5 & 0.005 & Chae et al. (2001) \\
\hline 9165 & 80 & 8.0 & 0.2 & 0.6 & 0.02 & Nindos and Zhang (2002) \\
\hline $\begin{array}{l}\text { Theoretical } \\
\text { upper bound }\end{array}$ & & & $\begin{array}{l}\leq 0.3 \\
\text { (shear only) }\end{array}$ & & $\leq 0.2$ & Démoulin et al. (2002b) \\
\hline $\begin{array}{l}\text { Flux tube } \\
\text { with N turns }\end{array}$ & & & $\mathrm{N}$ & & & \\
\hline
\end{tabular}

In most of the above-mentioned papers FWHM was chosen $\sim 8^{\prime \prime}$. The choice of $\Delta T$ is constrained by the characteristic horizontal velocities in the photosphere. During this $\Delta T$ time displacements (i) should be large enough to be well determined by the LCT, but at the same time (ii) they should be much smaller than the apodizing window size. Velocities $\geq 1-2 \mathrm{~km} \mathrm{~s}^{-1}$ are normally considered unphysical. In these published works $\Delta T$ was chosen to be 15 or 20 minutes.

The results are summarized in Table 1 . Helicity injected by photospheric footpoint motions and differential rotation during the analyzed periods are given in two units, one is the usual unit of $10^{42} \mathrm{Mx}^{2}$, the other is a "natural" unit of helicity expressed as the square of the total magnetic flux of the AR $\left(\Phi^{2}\right)$. Helicity expressed in this natural unit allows us to compare directly the results to a flux tube uniformly twisted in the cross section and having the same magnetic flux, i.e. the helicity of a flux rope having $\mathrm{N}$ number of turns is simply $\mathrm{N}$ in this natural unit. Expressing helicity in this way provides a convenient diagnostics even for the stability of the twisted flux tube, since simulations show that above a critical end-to-end twist in the range of 2.6-3.0 $\pi$ no equilibrium can be found, thus the rope ascends rapidly (Török and Kliem, 2002, and references therein). It is obvious from the values given in Table 1 that photospheric footpoint motions (during the intervals studied) do not provide enough helicity to bring the flux tube twist close to the critical value (1.3-1.5). This low efficiency can be due to the lack of a coherent pattern: areas where positive or negative helicity is injected co-exist at any time (Figure 2), significant shearing motions may last for a relatively short period during the lifetime of an AR (Figure 3), and may even reverse direction.

Active regions cover only a minor fraction of the solar surface. Quiet sun areas have low magnetic flux density, but they represent a huge amount of the total flux. Flux concentrations are in continuous motion following a random walk along random lattices (Lawrence and Schrijver, 1993). So it is important to find out how much helicity is injected by them. Using a tracking algorithm applied to high cadence ( $\sim 5$ minutes), high resolution (0.61"/pixel, smoothed over $\sim 3$ pixels) SOHO/MDI magnetograms, Welsch and Longcope (2003) obtained an injection rate of $\sim 5 \times 10^{19} \mathrm{Mx}^{2} \mathrm{~cm}^{-2} \mathrm{~s}^{-1}$ for the mutual magnetic helicity (the helicity coming from the winding of field lines from different flux elements about each other) in the quiet sun, which corresponds to a whole-cycle, hemispheric mutual helicity flux of $\sim 10^{42} \mathrm{Mx}^{2}$. The results of Welsch and Longcope (2003) indicate that the contribution of the quiet sun to helicity injection by footpoint motions is negligible.

However, it is well possible that these tracking methods, which have serious limitations, largely underestimate the amount of helicity injected by the shear term (Démoulin and Berger, 2003). One of the main limitations is low spatial resolution, which limits the obtained velocities to the velocity of group motion of the unresolved bunch of thin $(\leq 100 \mathrm{~km})$ flux tubes covered by a pixel. Also, tracking motions may have great difficulties in areas lacking significant contrast, like sunspot umbrae. The latter may lead to serious underestimation of the helicity flux, since most of the magnetic flux of the AR is located in such areas in 
the young active regions analysed (Table 1). Furthermore, motions along the isocontours of $\mathrm{B}_{n}$ (twisting) remain undetectable by these tracking methods. Another problem is linked to the $\Delta T$ and $w$ parameters of the LCT method, which prevent tracking of fast motions and have a smoothing effect on the velocities, because the correlated sub-parts, for which a mean velocity is derived, are typically 2-5 times larger than the spatial resolution of the magnetogram.

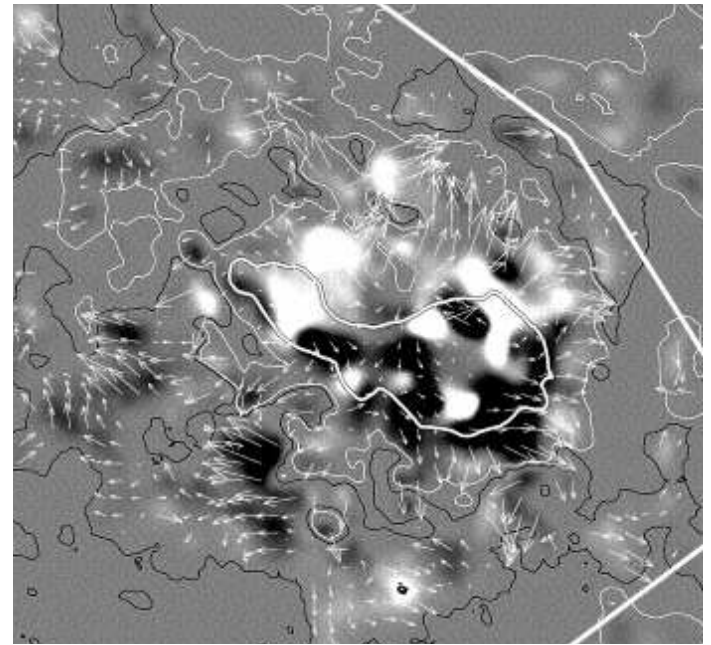

Fig. 2. Gray-scale map of helicity injection by horizontal footpoint motions, $-2\left(\vec{v} \cdot \vec{A}_{p}\right) B_{z}$, and the superposed transverse velocity vectors in active region NOAA 8668, as deduced from SOHO/MDI magnetograms using a local correlation tracking method. The contours indicate flux density levels of \pm 20 and $400 \mathrm{G}$. (Figure is used with permission, Chae et al., 2001). Note the patchiness of the gray-scale map: at the same time both negative and positive helicities are injected by footpoint motions. We propose that this mixed-sign pattern might be due to the effect of helical turbulence.
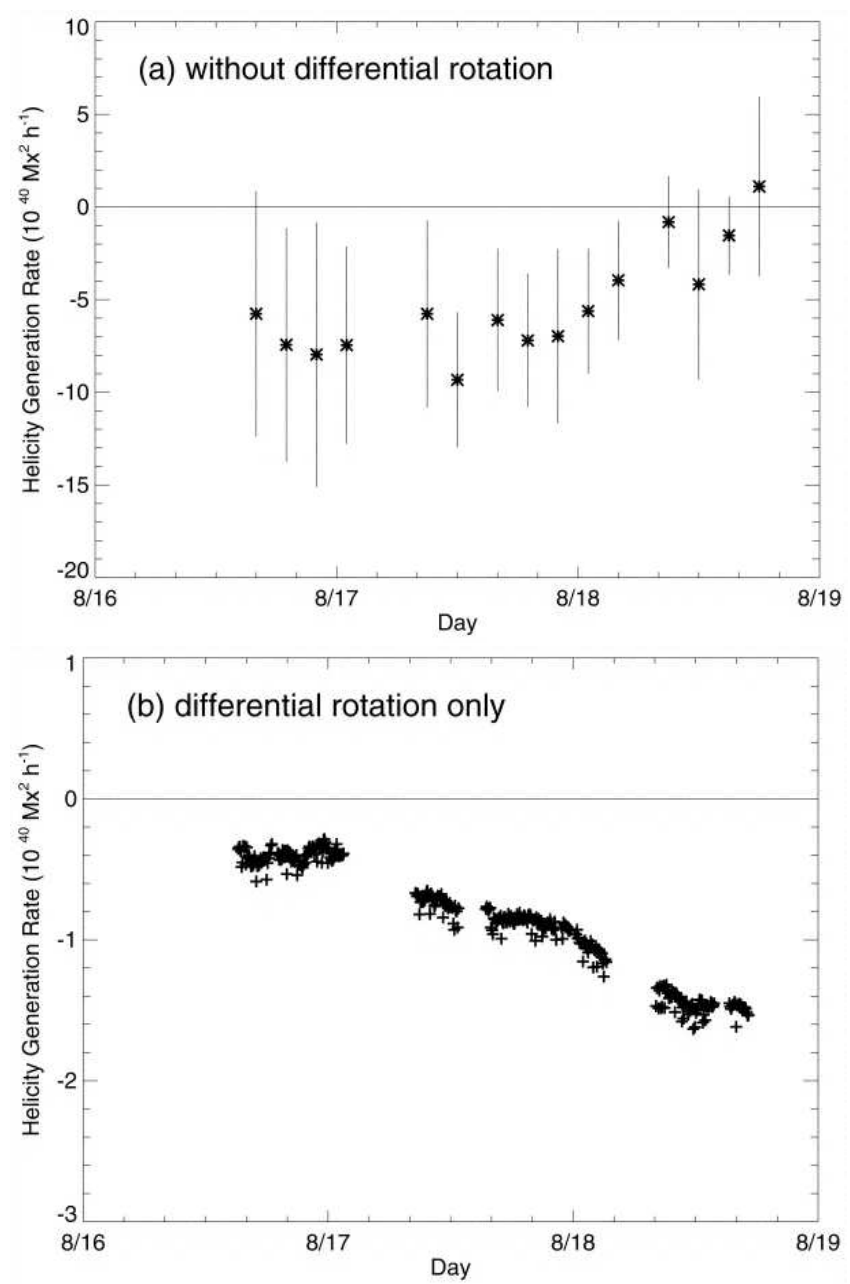

Fig. 3. Total helicity injection rate by photospheric footpoint motions and differential rotation in NOAA 8668 (Figures are used with permission, Chae et al., 2001). Note that the resultant helicity injection by footpoint motions decreases with time and that the contribution of differential rotation is minor.

Besides the problems with the velocity determination, there are problems with the determination of $\mathrm{B}_{n}$ as well. It was discovered recently that $\mathrm{SOHO} / \mathrm{MDI}$ underestimates magnetic flux densities by a factor of 0.64-0.69 (Berger and Lites, 2002), which means that all the numbers in Table 1 have to be multiplied by a factor of $\sim 2$. However, in areas with high magnetic flux density $(\geq 1300 \mathrm{G})$, the response of MDI becomes non-linear, which amplifies the underestimation of the helicity flux in strong umbrae. A more detailed discussion on the possible errors leading to underestimation of helicity injected by the shear term is given by Démoulin and Berger (2003).

\section{Computations of the Advection Term}

Computations of the advection term $2 \int_{S}\left(\overrightarrow{A_{0}} \cdot \vec{B}\right) \vec{v} \cdot \overrightarrow{d S}$ from observations involves measurements of the transverse components of the magnetic field and longitudinal velocities, from which $\mathrm{B}_{t}$ and $\mathrm{v}_{n}$ can be determined. Vector magnetograms (after calibration and removal of the $180^{\circ}$ ambiguity) can be transformed to provide photospheric maps of $\mathrm{B}_{n}$ and $\mathrm{B}_{t}$. Longitudinal velocities are classically derived from the Doppler shift of spectral lines. However, since there is no safe way to transform them to $\mathrm{v}_{n}$, they only provide useful data 
at disc center. Furthermore, in emerging magnetic field regions, where the advection term should be the largest, both upflows and downflows are present (convective collapse!), and the downflows may be 2-5 times stronger than upflows (e.g. Lites et al., 1998, and references therein); therefore there is no safe way to deduce $\mathrm{v}_{n}$ from Doppler measurements.

The first quantitative estimate of the helicity injection into an observed solar active region was made by Wang (1996), who deduced that $10^{43} \mathrm{Mx}^{2}$ of helicity was produced in an emerging flux region (AR 6233) over a period of just a few hours. He computed change in magnetic helicity density using vector magnetograms and tracing the change of $\alpha$, the force-free parameter.

Kusano et al. (2002) made an attempt to derive helicity flux through the photosphere in active region NOAA 8100 by using observations of $\vec{B}$ and $v_{t}$ (using a correlation tracking method) and computing $\mathrm{v}_{n}$ from the induction equation. Their results show that both photospheric shear motions and flux emergence injected magnetic helicity into this active region, and that these two terms supplied helicity of opposite signs.

However, a correlation tracking method is not measuring the plasma motions, but rather the displacement of the photospheric cuts of magnetic flux tubes. Such displacement is the result of both horizontal and vertical plasma motions. Démoulin \& Berger (2002) show that both the shear and advection terms can be combined in one single term, in which the displacement velocity is used. More precisely, using the velocity deduced from a correlation tracking method, we get the full helicity flux (within the limitations of the observations). Thus, Démoulin \& Berger (2002) show that it is not correct to add the helicity flux associated to vertical plasma motions to the helicity flux deduced from the velocities of photospheric footpoint motions.

\section{Computations of Relative Magnetic Helicity in the Corona}

Démoulin et al. (2002a) and Green et al. (2002) worked out a method to compute coronal relative magnetic helicity from observations. They used SOHO/MDI magnetograms taken close to the central meridian passages of the studied ARs as boundary conditions for linear force-free field (lfff) magnetic extrapolations $(\vec{\nabla} \times \vec{B}=\alpha \vec{B} ; \alpha=$ const $)$. The extrapolated field lines were fitted with coronal loops observed with Yohkoh/SXT. The $\alpha$ value giving the best general fit between the models and observations was adopted for computation of the coronal helicity following Berger (1985): $H_{r}=2 \alpha \sum_{n_{x}=1}^{N_{x}} \sum_{n_{y}=1}^{N_{y}}\left|\widetilde{B}_{n_{x}, n_{y}}^{2}\right| / l\left(k_{x}^{2}+k_{y}^{2}\right)$, where $\widetilde{B}_{n_{x}, n_{y}}$ is the Fourier amplitude of the field component $B_{n}, l=\sqrt{k_{x}^{2}+k_{y}^{2}-\alpha^{2}}, k_{x}=2 \pi n_{x} / L, k_{y}=2 \pi n_{y} / L$ with $L$ being the horizontal extension of the computational box.

Coronal magnetic helicity content of an AR depends on the photospheric flux distribution and on the value of $\alpha$. Even though $\alpha$ stays in all their extrapolations below its resonant value (which would give unrealistically high magnetic helicity), these authors used a linearized expression in $\alpha$ for the helicity computations (see Green et al. 2002). Since they analysed the evolution of coronal magnetic helicity in ARs as they evolved, it was important to keep the computational box of the same extension centered on the AR in all the cases. These computations of coronal relative magnetic helicity (Démoulin et al., 2002a; Green et al., 2002) carry the problems of the lfff models, which are imperfect representations of the coronal field. However, the order of magnitude obtained for large ARs $\left(5 \times 10^{42}-2 \times 10^{43} \mathrm{Mx}^{2}\right)$ agrees quite well with theoretical expectations, thus they appear to be reasonably good estimates.

Régnier et al. (2002) utilized IVM vector magnetograms and a non-linear force-free field model to reconstruct the coronal field of a sigmoidal AR (NOAA 8151). Computing relative coronal magnetic helicity from their model, these authors obtained a value of $4.7 \times 10^{34} \mathrm{Mx}^{2}$, which, being eight orders of magnitude lower than the estimate for the helicity of any reasonably sheared coronal field and for the helicity carried away by a single CME of average size, seems to be far from being realistic.

\section{Magnetic Helicity Ejected via CMEs}

A CME is the result of the instability of the coronal field, so it will carry away part of the magnetic helicity of its source magnetic field. There is increasing evidence indicating that the helicity sign of MCs matches that of their source region, i.e. their associated erupting filament (Bothmer and Schwenn, 1994; Rust, 1994; Marubashi, 1997; Yurchyshyn et al., 2001). Assuming a one to one correspondence between CMEs and MCs, i.e. interplanetary twisted flux tubes (e.g. Webb et al., 2000), the helicity ejected in a CME can be evaluated using "in situ" measurements of the interplanetary magnetic field. Démoulin et al. (2002a) computed the 

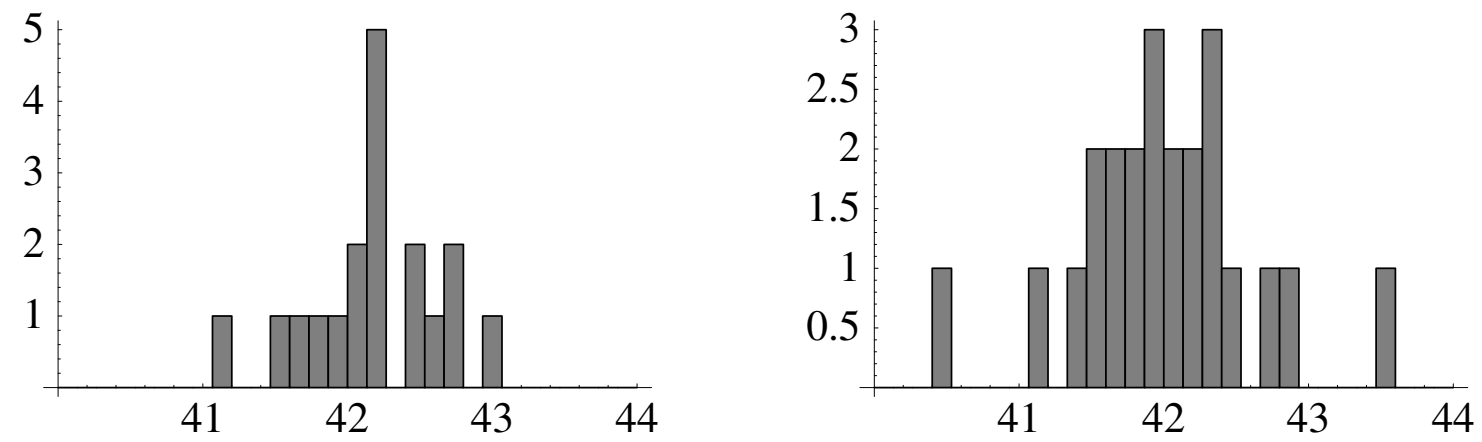

Fig. 4. Distribution of the relative magnetic helicity of magnetic clouds (MCs) calculated from mean $B_{0}$ (axial magnetic field strength) and R (radius) measured in two samples of MCs by Lepping et al. (1990; $18 \mathrm{MCs}$, left panel) and Zhao et al. (2001, $23 \mathrm{MCs}$, right panel). The two samples overlap, since 11 clouds are the same in both studies, though they use different fitting models (in the newer sample the expansion of the flux tube during spacecraft crossing is taken into account). The mean relative magnetic helicity values are $2.4 \times 10^{42} \mathrm{Mx}^{2}$ and $2.8 \times 10^{42} \mathrm{Mx}^{2}$, respectively, assuming a flux tube length of $0.5 \mathrm{AU}$.

relative helicity per unit length in a twisted interplanetary flux tube using a numerically integrated form of Berger's (1999) equation, and the average values of the axial magnetic field $B_{0}\left((2.0 \pm 0.7) \times 10^{-4} \mathrm{G}\right)$ and radius $\mathrm{R}\left((2.1 \pm 0.7) \times 10^{12} \mathrm{~cm}\right)$ for a set of 18 clouds studied by Lepping et al. (1990). The same computation was done for another sample of 23 clouds analyzed by Zhao et al. $(2001)\left(B_{0}=(2.4 \pm 0.8) \times 10^{-4} \mathrm{G}\right.$ and $\left.\mathrm{R}=(1.7 \pm 0.8) \times 10^{12}\right)$. For the length of the interplanetary flux tube two values were used: $L_{1}=0.5 \mathrm{AU}$ (DeVore, 2000) which yielded $H_{r} \approx 2 \times 10^{42} \mathrm{Mx}^{2}$, and $L_{2}=2 A U$ (the cloud is still connected to the Sun; see e.g. Richardson, 1997) which gives four times as much helicity for one average-sized MC, i.e. CME. These mean helicity values have to be multiplied by the number of the CMEs to obtain the total magnetic helicity ejected from an AR.

During the entire lifetime of two ARs (6 and 5 solar rotations, respectively) such counts have been made: NOAA 7978 was found to be the source region of 31 CMEs (Démoulin et al., 2002a), while NOAA 8100 produced 65 CMEs (Green et al., 2002). These numbers include corrections for data gaps and far-side locations of the ARs. They are much higher than previously thought based on pre-SOHO observations (4-5/AR/lifetime; e.g. DeVore, 2000), and imply a huge amount of (unsigned) helicity ejected from these ARs into the interplanetary space: $62-248 \times 10^{42} \mathrm{Mx}^{2}$ (AR 7978) and $130-520 \times 10^{42} \mathrm{Mx}^{2}(\mathrm{AR} 8100)$, where the lower/upper estimates imply flux tube length of $0.5 / 2 \mathrm{AU}$.

Nindos and Zhang (2002) studied a MC which was linked to a CME originating in AR 9165, in which they studied helicity injection by photospheric motions. Using the method of Démoulin et al. (2002b) and $\mathrm{B}_{0}$ and $\mathrm{R}$ measurements for this $\mathrm{MC}$, the magnetic helicity was found as large as 32 (resp. 127) $\times 10^{42} \mathrm{Mx}^{2}$ with a flux tube length of 0.5 (resp. 2) AU ! However, taking the average of MCs observed in the year 2000 they obtained an average magnetic helicity per cloud of 3.9 (resp. 15.4) $\times 10^{42} \mathrm{Mx}^{2}$, about a factor 2 above the mean values of the data shown in Figure 4.

There is a very large uncertainty in these helicity numbers, thus they should be regarded as rough approximative values. The greatest unknown is the length of the flux tube in a MC. Furthermore, even well-observed MCs, like the ones contained in the two samples used for the computations, show a great spread (Figure 4). Note that magnetic helicity depends on the third power of $R$ and the second power of $B_{0}$, so their spread is amplified in the helicity results. Currently, we do not know the distribution of twist along the interplanetary flux rope, whether it is uniform, or concentrated in its front or its legs, which adds to the uncertainties.

\section{Magnetic Helicity Budget of Active Regions}

The total helicity budget of an active region may be written: 
$H_{\text {flux from photospheric motions }}=\Delta H_{\text {corona }}+N \cdot H_{\mathrm{CME}}$, where $\Delta$ denotes the variation of the helicity, $\mathrm{N}$ is the number of the CMEs and $H_{\mathrm{CME}}$ is the mean helicity carried away per CME event.

Three groups have drawn the helicity budget of ARs, deriving from observations: (i) the helicity injection at the photospheric level by differential rotation and/or local footpoint motions, (ii) the changes in the coronal helicity, as well as (iii) the helicity carried away by CMEs (Démoulin et al., 2002a; Green et al., 2002; Nindos and Zhang, 2002).

Their results are in good accordance: (i) helicity injected at the photospheric level by differential rotation is a minor contributor to the global helicity budget: CMEs remove at least 10 times more helicity than the one injected by differential rotation; (ii) photospheric shearing motions may inject much more helicity than the differential rotation, but present studies are limited to few days and also by the spatial resolution of observations among other problems; (iii) consequently the main source of magnetic helicity carried away in CMEs is still undetected at the photospheric level.

For example, for NOAA 7978 which was a simple isolated bipolar AR, Démoulin et al. (2002a) found that an amount of $\Delta H=49-235 \times 10^{42} \mathrm{Mx}^{2}$ of magnetic helicity should be provided to the corona in order to account for all the CMEs. Expressing this in the "natural" units of helicity, i.e. in the square of the total magnetic flux of the AR, we obtain 0.25-1 $\Phi^{2}$, since the magnetic flux of AR 7978 was $\Phi \sim 1.5 \times 10^{22} \mathrm{Mx}$ (note that all these numbers are corrected for flux underestimation by MDI as derived by Berger and Lites, 2002). Since helicity expressed in this natural unit reveals the number of turns a flux rope has end-to-end, it actually shows that 31 CMEs carried away the equivalent helicity contained in the flux tube forming the AR having about one turn (if uniformly twisted across the flux tube).

This total helicity appears to be consistent with previous theoretical results. Moreno-Insertis and Emonet (1996), Emonet and Moreno-Insertis (1998) showed that flux tubes, to be able to survive their rise through the convection zone, need to be twisted by a few turns. Though recent simulations by Abbett et al. (2001) indicated that the Coriolis force may also have a stabilizing effect on rising flux tubes, the need for them to be twisted did not disappear. Thus the twist obtained from the helicity budgets seems to be in the right range.

\section{HOW TO GET BETTER MEASUREMENTS OF MAGNETIC HELICITY?}

Direct observations, like helioseismology, do not seem to be able to put constraints on sub-surface generation of magnetic helicity in the near future. However, hopes are raised by the recent development of dynamo theories which are incorporating the analysis (and constraint!) of magnetic helicity (e.g. Brandenburg, 2001). Moreover, a combination of theoretical advances in simulations of thin flux tubes and better photospheric observations will be able to advance our knowledge on flux tubes in the convective zone, in particular on their twist/writhe magnetic helicity.

Improving photospheric measurements of $\vec{B}$ and $\vec{v}$ are crucial for a more precise determination of the helicity fluxes. High resolution vector magnetographs, like ASP, THEMIS and SOLAR B will provide suitable data for that.

For a better determination of coronal helicity we need improved magnetic field extrapolations and high resolution multi-wavelength coronal loop observations.

For better heliospheric helicity estimates we need more realistic models of MCs, and to advance our knowledge on the length of field lines in MCs (to be able to trace field lines, e.g. Larson et al., 1997). It would also be important to find out how uniform the twist is along an interplanetary twisted flux tube, which we may be able to measure if the same MC would pass by STEREO A and B.

For an improved link between helicity in the solar corona and heliosphere we have to associate a given CME to a given MC, which will have a better chance when STEREO and its SECCHI heliospheric imager are operational. Finally, we need to combine global and local measurements to achieve our goal of having a complete budget of magnetic helicity. After that, we will be in position to tackle the more difficult problem of the energy budget.

\section{ACKNOWLEDGEMENTS}

L.v.D.G. is supported by the Research fellowship grant F/02/035 of the K.U. Leuven and by the Hungarian 
Government grants OTKA T-038013, and T-032846. P.D. and L.v.D.G. acknowledge the French-Hungarian Science and Technology cooperative program. P.D. and C.H.M. acknowledge financial support from ECOS (France) and SECyT (Argentina) through their cooperative science program (A01U04).

\section{REFERENCES}

Abbett, W.P., G.H. Fisher, Y. Fan, The effects of rotation on the evolution of rising $\Omega$ loops in a stratified model convection zone, Astrophys. J., 546, 1194-1203, 2001.

Abramenko, V.I., T. Wang, and V.B. Yurchishin, Analysis of electric current helicity in active regions on the basis of vector magnetograms, Sol. Phys., 168, 75-89, 1996.

Berger, M.A., Rigorous new limits on magnetic helicity dissipation in the solar corona, Geophys. Astrophys. Fluid. Dynamics, 30, 79-104, 1984.

Berger, M.A., Structure and stability of constant-alpha force-free fields, Astrophys. J. Suppl. Ser., 59, 433-444, 1985.

Berger, M.A., Topological invariants of field lines rooted to planes, Geophys. Astrophys. Fluid. Dynamics, 34, 265-281, 1986.

Berger, M.A., An energy formula for nonlinear force-free magnetic fields, Astron. Astrophys., 201, 355-361, 1988.

Berger, M.A., Magnetic helicity in space physics, in Magnetic Helicity in Space and Laboratory Plasmas, edited by M.R. Brown, R.C. Canfield, and A.A. Pevtsov, Geophys. Monograph 111, A.G.U., 1-9, 1999.

Berger, M.A., and G.B. Field, The topological properties of magnetic helicity, J. Fluid. Mech., 147, 133-148, 1984.

Berger, M.A., and A. Ruzmaikin, Rate of helicity production by solar rotation, J. Geophys. Res., 105, A5, 10481-10490, 2000.

Berger, T.E., and B.W. Lites, Weak field magnetogram calibration using Advanced Stokes Polarimeter flux density maps: II. SOHO/MDI full-disk mode calibration, submitted to Sol. Phys., 2002.

Bothmer, V., and R. Schwenn, Eruptive prominences as sources of magnetic clouds in the solar wind, Space Sci. Rev., 70, 215-220, 1994.

Brandenburg, A., The inverse cascade and nonlinear $\alpha$-effect in simulations of isotropic helical hydromagnetic turbulence, Astrophys. J., 550, 824-840, 2001.

Brandenburg, A., and E.G. Blackman, How magnetic helicity ejection can speed up large scale dynamos, Adv. Space Res., in this issue, 2003.

Canfield, R.C., H.S. Hudson, and D.E. McKenzie, Sigmoidal morphology and eruptive solar activity, Geophys. Res. Lett., 26, 627-630, 1999.

Chae, J., 2001, Observational determination of the rate of magnetic helicity transport through the solar surface via the horizontal motion of field line footpoints, Astrophys. J., 560, L95-L98, 2001.

Chae, J., H. Wang, J. Qiu, et al., The formation of a prominence in active region NOAA 8668. I. SOHO/MDI observations of magnetic field evolution, Astrophys. J., 560, 476-489, 2002.

Démoulin P., and M.A. Berger, Magnetic energy and helicity fluxes at the photospheric level, submitted to Sol. Phys., 2002.

Démoulin P., C.H. Mandrini, L. van Driel-Gesztelyi, et al., What is the source of the magnetic helicity shed by CMEs? The long-term helicity budget of AR 7978, Astron. Astrophys., 382, 650-665, 2002a.

Démoulin P., C.H. Mandrini, L. van Driel-Gesztelyi, et al., The magnetic helicity injected by shearing motions, Sol. Phys., 207, 87-110, 2002b.

DeVore, C.R., Magnetic Helicity Generation by Solar Differential Rotation, Astrophys. J., 539, 944-953, 2000.

Emonet, T., F. Moreno-Insertis, The physics of twisted magnetic tubes rising in a stratified medium: twodimensional results, Astrophys. J., 492, 804-821, 1998.

Green L.G., M.C. López-Fuentes, C.H. Mandrini, et al., The magnetic helicity budget of a CME-prolific active region, Sol. Phys., 208, 43-68, 2002.

Hale, G.E., Nature of the hydrogen vortices surrounding sun-spots, Publ. Astron. Soc. Pacific, 37, 268-270, 1925.

Kusano, K., T. Maeshiro, T. Yokoyama, et al., Measurement of magnetic helicity injection and free energy 
loading into the solar corona Astrophys. J., 577, 501-512, 2002.

Larson, D.E., R.P. Lin, J.M. McTiernan, et al., Tracing the topology of the October 18-20, 1995, magnetic cloud with 0.1-102 keV electrons, Geophys. Res. Let., 24, 1911-1914, 1997.

Lawrence, J.K., and C.J. Schrijver, Anomalous diffusion of magnetic elements across the solar surface, Astrophys. J., 411, 402-405, 1993.

Lepping, R.P., L.F. Burlaga, and J.A. Jones, Magnetic field structure of interplanetary magnetic clouds at 1 AU, J. Geophys. Res., 95, 11957-11965, 1990.

Leroy, J.L., V. Bommier, and S. Sahal-Bréchot, The magnetic field in the prominences of the polar crown, Sol. Phys., 83, 135-142, 1983.

Lites, B.W., A. Skumanich, and V. Martínez Pillet, Vector magnetic fields of emerging solar flux. I. Properties at the site of emergence, Astron. Astrophys., 333, 1053-1068, 1998.

Longcope, D.W., and A.A. Pevtsov, Helicity transport and creation in the solar convection zone, Adv. Space Res., in this issue, 2003.

Marubashi, K., Interplanetary magnetic flux ropes and solar filament, in Coronal mass ejections, edited by N. Crooker, J.A. Joselyn, and J. Feynman, Geophys. Monograph 99, A.G.U., 147-156, 1997.

Martin, S.F., R. Bilimoria, and P.W. Tracadas, Magnetic field configurations basic to filament channels and filaments, in Solar Surface Magnetism, edited by R.J. Rutten and C.J. Schrijver, Dordrecht, Kluwer, 303-338, 1994.

Martin, S.F., and A.H. McAllister, Predicting the sign of magnetic helicity in erupting filaments and coronal mass ejections, in Coronal Mass Ejections, edited by N. Crooker, J. A. Joselyn, and J. Feynman, Geophys. Monograph 99, A.G.U., 127-138, 1997.

Moon, Y.-J., J. Chae, G.S. Choe, et al., Flare activity and magnetic helicity injection by photospheric horizontal motions, Astrophys. J., 574, 1066-1073, 2002.

Moreno-Insertis, F., and T. Emonet, The rise of twisted magnetic tubes in a stratified medium, Astrophys. J., 472, L53-L56, 1996.

Nakagawa, Y., M.A. Raadu, D.E. Billings, et al., On the topology of filaments and chromospheric fibrils near sunspots, Sol. Phys., 19, 72-85, 1971.

Nindos, A., and H. Zhang, Photospheric motions and coronal mass ejection productivity, Astrophys. J., 573, L133-L136, 2002.

November, L.J., and G.W. Simon, Precise proper-motion measurement of solar granulation, Astrophys. J., 333, 427-442, 1988.

Peter, H., Superpenumbral vortices, Mon. Not. Royal Astron. Soc., 278, 821-828, 1996.

Pevtsov, A.A., Helicity patterns on the Sun, Adv. Space Res., in this issue, 2003.

Pevtsov, A.A., R.C. Canfield, and T.R. Metcalf, Latitudinal variation of helicity of photospheric magnetic fields, Astrophys. J., 440, L109-L112, 1995.

Régnier, S., T. Amari, and E. Kersalé, 3D Coronal magnetic field from vector magnetograms: non-constantalpha force-free configuration of the active region NOAA 8151, Astron. Astrophys., 392, 1119-1127, 2002.

Richardson, I.G., Using energetic particles to probe the magnetic topology of ejecta, in Coronal Mass Ejections, in Coronal Mass Ejections, edited by N. Crooker, J. A. Joselyn, and J. Feynman, Geophys. Monograph 99, A.G.U., 189-196, 1997.

Richardson, R.S., The nature of solar hydrogen vortices, Astrophys. J., 93, 24-28, 1941.

Rust, D.M., Spawning and shedding helical magnetic fields in the solar atmosphere, Geophys. Res. Lett., 21, 241-244, 1994.

Rust, D.M., and A. Kumar, Evidence for helically kinked magnetic flux ropes in solar eruptions Astrophys. J., 464, L199-L202, 1996.

Seehafer, N., Electric current helicity in the solar atmosphere, Sol. Phys., 125, 219-232, 1990.

Seehafer, N., Helicity and the solar dynamo, Adv. Space Res., in this issue, 2003.

Török, T., and B. Kliem, The evolution of twisting coronal magnetic flux tubes, submitted to Astron. Astrophys., 2002.

Wang, J., A Note on the evolution of magnetic helicity in active regions, Sol. Phys., 63, 319-325, 1996.

Webb, D.F., E W. Cliver, N.U. Crooker, et al., Relationship of halo coronal mass ejections, magnetic clouds, and magnetic storms, Geophys. Res. Let., 105, A4, 7491-7508, 2000. 
Welsch, B.T., and D.W. Longcope, Magnetic helicity injection by horizontal flows in the quiet Sun: I. Mutual helicity flux, Astrophys. J., in press, 2003.

Yurchyshyn, V.B., H. Wang, P.R. Goode, and Y. Deng, Orientation of the magnetic fields in interplanetary flux ropes and Solar Filaments, Astrophys. J., 563, 381-388, 2001.

Zhao, X.P., J.T. Hoeksema, and K. Marubashi, Magnetic cloud $\mathrm{B}_{s}$ events and their dependence on cloud parameters, J. Geophys. Res., 106, 15643-15656, 2001.

e-mail addresses:

Lidia.vanDriel@obspm.fr, Pascal.Demoulin@obspm.fr, mandrini@iafe.uba.ar

Manuscript received

; revised

; accepted 